Supporting computational data for

\title{
A Highly Reactive Ruthenium Phosphido Complex Exhibiting Ru-P $\pi$-Bonding
}

Eric J. Derrah, Dimitrios A. Pantazis ${ }^{*}$, Robert McDonald ${ }^{\ddagger}$, and Lisa Rosenberg* ${ }^{*}$

Department of Chemistry, University of Victoria, P.O. Box 3065, Victoria, British Columbia, Canada, V8W 3 V6

${ }^{*}$ WestCHEM, Department of Chemistry, University of Glasgow, Glasgow, United Kingdom, G12 8QQ

${ }^{7}$ X-ray Crystallography Laboratory, Department of Chemistry, University of Alberta, Edmonton, Alberta, Canada, T6G $2 G 2$

\section{$\underline{\text { Results from DFT and TD-DFT calculations }}^{1}$}

Table 1. Total energy (in hartrees) and optimized cartesian coordinates (in $\AA$ ) for $\mathbf{2 a}$

$\mathrm{E}(\mathrm{RPBE}+\mathrm{HF}-\mathrm{PBE})=\quad$ 2274.40197162

\begin{tabular}{|c|c|c|c|}
\hline 44 & 0.0863360 & $Z^{1.1067690}$ & - 0.4148330 \\
\hline 15 & ${ }_{-1.7824780}$ & _o.1269230 & 0.0724260 \\
\hline 15 & 1.4106070 & 0.7022570 & _0.0849400 \\
\hline 6 & -0.5712350 & ${ }_{-3.1396100}$ & _0.9921710 \\
\hline 1 & -1.5844230 & -3.5181530 & -0.9435560 \\
\hline 6 & 0.0296060 & -2.4734100 & -2.1055490 \\
\hline 1 & -0.4630210 & ${ }^{2} .2239270$ & $z_{3}^{3.0406630}$ \\
\hline 6 & 1.3995840 & -2.2089440 & -1.7953760 \\
\hline 1 & 2.1218050 & ${ }^{1} 1.7604870$ & $z^{2} .4671690$ \\
\hline 6 & 1.6863710 & ${ }^{2} .8037720$ & 0.5102360 \\
\hline 6 & 2.8769940 & ${ }^{2} .9361890$ & 0.2473940 \\
\hline 1 & 3.8188310 & $\_^{2} .5828170$ & -0.1605360 \\
\hline 6 & 2.8280170 & -3.5787350 & 1.4633990 \\
\hline 1 & 3.7422700 & ${ }_{-3.7084560}$ & 2.0409250 \\
\hline 6 & 1.6052230 & ${ }^{4} .0779030$ & 1.9833390 \\
\hline 1 & 1.6024110 & ${ }_{-4.5679730}$ & 2.9561930 \\
\hline 6 & 0.4296030 & -3.9629280 & 1.2779460 \\
\hline 1 & -0.5047770 & -4.3614150 & 1.6715490 \\
\hline 6 & 0.4501970 & -3.3468400 & _0.0002090 \\
\hline 6 & $z^{2} .1624440$ & 1.6824530 & 0.3224770 \\
\hline 1 & ${ }^{1} 1.1730660$ & 2.1752450 & 0.3237670 \\
\hline 6 & ${ }^{2} .8278930$ & 1.9526980 & 1.7012770 \\
\hline 1 & ${ }_{-3.8039290}$ & 1.4446290 & 1.7514340 \\
\hline 1 & ${ }^{2} .1906910$ & 1.5456160 & 2.5024860 \\
\hline 6 & ${ }_{-3.0313860}$ & 3.4736550 & 1.9161360 \\
\hline 1 & -3.5355700 & 3.6448740 & 2.8791960 \\
\hline 1 & $Z^{2} .0444150$ & 3.9610840 & 1.9654760 \\
\hline 6 & ${ }_{-3.8573600}$ & 4.0966850 & 0.7672600 \\
\hline 1 & -4.8724100 & 3.6693760 & 0.7748320 \\
\hline 1 & -3.9542830 & 5.1818290 & 0.9225370 \\
\hline 6 & -3.1976180 & 3.8189660 & -0.6031230 \\
\hline 1 & $\_^{2} .2153510$ & 4.3170740 & -0.6464220 \\
\hline 1 & -3.8176970 & 4.2347570 & -1.4116210 \\
\hline 6 & ${ }_{-3}^{3.0018960}$ & 2.2989540 & -0.8295690 \\
\hline 1 & $\_^{2} .4916640$ & 2.1293410 & ${ }_{-1.7917420}$ \\
\hline 1 & -3.9881830 & 1.8117800 & -0.8767810 \\
\hline 6 & -3.4708870 & -0.9146070 & -0.0628770 \\
\hline 1 & ${ }^{4} .2176070$ & -0.1513230 & 0.2260960 \\
\hline 6 & -3.5922810 & $z^{2} .0986260$ & 0.9310150 \\
\hline
\end{tabular}




\begin{tabular}{|c|c|c|}
\hline$z^{2} .7845820$ & $ـ^{2} .8249230$ & 0.7259760 \\
\hline-3.4430340 & 1.7304520 & 1.9590920 \\
\hline-4.9651540 & -2.8028590 & 0.8089420 \\
\hline-4.9958860 & -3.6662450 & 1.4902070 \\
\hline-5.7597050 & -2.1050030 & 1.1179150 \\
\hline-5.2249210 & -3.2624570 & -0.6433200 \\
\hline${ }_{-}^{4} .4694580$ & ${ }^{4} .0146150$ & _o.9295440 \\
\hline-6.2119390 & -3.7440140 & -0.7148560 \\
\hline${ }_{-5.1492270}$ & $z^{2} .0662810$ & ${ }_{-1.6182820}$ \\
\hline-5.9477390 & -1.3467840 & -1.3764350 \\
\hline${ }_{-5.3096260}$ & $ـ^{2} .4087330$ & $ـ^{2.6515610}$ \\
\hline-3.7761820 & -1.3576350 & -1.5183280 \\
\hline-3.7523810 & _0.4799500 & $ـ^{2} .1846900$ \\
\hline$z^{2} .9821880$ & $z^{2} .0482620$ & ${ }_{-1.8574080}$ \\
\hline 1.1713120 & 1.4914520 & ${ }^{-} 1.5466990$ \\
\hline 0.9173020 & 0.6013270 & 2.6219270 \\
\hline 0.8669940 & _0.4782990 & 2.4179160 \\
\hline 0.6937950 & 1.0954760 & 3.9156130 \\
\hline 0.4982990 & 0.3965700 & 4.7378720 \\
\hline 0.7091020 & 2.4794600 & 4.1489260 \\
\hline 0.5252360 & 2.8673410 & 5.1573200 \\
\hline 0.9513200 & 3.3665730 & 3.0906030 \\
\hline 0.9585450 & 4.4469560 & 3.2722600 \\
\hline 1.1876270 & 2.8793920 & 1.7966020 \\
\hline 1.3711670 & 3.5836580 & 0.9784690 \\
\hline 1.3423540 & 2.0137910 & ${ }_{-1.3526270}$ \\
\hline 0.4228130 & 1.8539780 & -2.4132700 \\
\hline _-0.2232890 & 0.9640740 & -2.4082510 \\
\hline 0.3581470 & 2.8019230 & ${ }_{-3.4478530}$ \\
\hline-0.3583480 & 2.6669610 & -4.2671330 \\
\hline 1.2140740 & 3.9114890 & -3.4341470 \\
\hline 1.1653130 & 4.6525460 & -4.2403680 \\
\hline 2.1485970 & 4.0668660 & $ـ^{2.3977740}$ \\
\hline 2.8303480 & 4.9244820 & $-^{2} .3991000$ \\
\hline 2.2214160 & 3.1213970 & $n^{1.3671360}$ \\
\hline 2.9774410 & 3.2330860 & -0.5806640 \\
\hline 3.1797210 & 0.2524490 & -0.1063810 \\
\hline 3.8217580 & 0.0699390 & -1.3575910 \\
\hline 3.2864150 & 0.3199720 & $\_^{2.2829420}$ \\
\hline 5.1381790 & _0.4091510 & ${ }_{-1.4187780}$ \\
\hline 5.6228110 & _0.5434750 & -2.3927710 \\
\hline 5.8363850 & _0.7007940 & -0.2355030 \\
\hline 6.8663330 & $ـ^{1.0717000}$ & -0.2847040 \\
\hline 5.2180180 & _0.5066840 & 1.0087040 \\
\hline 5.7643210 & _0.7215520 & 1.934132 \\
\hline 3.8969620 & _0.0391840 & 1.076770 \\
\hline 3.4200390 & 0.1012840 & 2.054072 \\
\hline
\end{tabular}


Table 2. Energies, oscillator strengths and molecular orbital assignments for the ten lowest TD-DFT calculated excitations.

\begin{tabular}{|c|c|c|}
\hline$E, \mathrm{~nm}(\mathrm{eV})$ & $f$ & MO transitions $(\mathrm{H}=\mathrm{HOMO}, \mathrm{L}=\mathrm{LUMO})$ \\
\hline $714(1.73)$ & 0.001 & H_1 $\rightarrow$ L $(83 \%)$ \\
\hline $600(2.06)$ & 0.024 & $\mathrm{H}^{-} \rightarrow \mathrm{L}(67 \%), \mathrm{H} \_2 \rightarrow \mathrm{L}(8 \%)$ \\
\hline $439(2.82)$ & 0.005 & H_3 $\rightarrow$ L $(63 \%),{ }_{1} \_2 \rightarrow \mathrm{L}(13 \%)$ \\
\hline $390(3.18)$ & 0.008 & $\mathrm{H}_{-} 1 \rightarrow \mathrm{L}+1(49 \%), \mathrm{H} \rightarrow \mathrm{L}+1(25 \%), \mathrm{H}_{2} 2 \rightarrow \mathrm{L}(8 \%)$ \\
\hline $378(3.28)$ & 0.017 & $\mathrm{H} \rightarrow \mathrm{L}+1(50 \%), \mathrm{H} \_1 \rightarrow \mathrm{L}+1(26 \%)$ \\
\hline $352(3.52)$ & 0.139 & H_2 $\rightarrow$ L $(55 \%), H \_4 \rightarrow \mathrm{L}(10 \%), \mathrm{H} \_3 \rightarrow \mathrm{L}(9 \%)$ \\
\hline $330(3.76)$ & 0.064 & $\mathrm{H}_{-} 4 \rightarrow \mathrm{L}(41 \%), \mathrm{H} \rightarrow \mathrm{L}+2(18 \%), \mathrm{H}-0 \rightarrow \mathrm{L}+3(18 \%)$ \\
\hline $322(3.85)$ & 0.024 & $\mathrm{H}_{-} 1 \rightarrow \mathrm{L}+2(33 \%), \mathrm{H} 1 \rightarrow \mathrm{L}+3(28 \%), \mathrm{H} \rightarrow \mathrm{L}+2(16 \%)$ \\
\hline $313(3.97)$ & 0.017 & $\mathrm{H} \rightarrow \mathrm{L}+2(57 \%), \mathrm{H} \_4 \rightarrow \mathrm{L}(13 \%), \mathrm{H} \_1 \rightarrow \mathrm{L}+2(8 \%), \mathrm{H} \_1 \rightarrow \mathrm{L}+3(7 \%)$ \\
\hline $304(4.08)$ & 0.002 & $\mathrm{H} 33 \rightarrow \mathrm{L}+1(26 \%), \bar{H} 1 \rightarrow \mathrm{L}+2(25 \%), \mathrm{H} \rightarrow \mathrm{L}+3(15 \%), \mathrm{H} \_2 \rightarrow \mathrm{L}+1(10 \%)$ \\
\hline
\end{tabular}

Table 3. Total energies (in hartrees) and optimized cartesian coordinates (in $\AA$ ) for the simplified models of species $\mathbf{2}$ and $\mathbf{3}$, and the transition state for their interconversion.

Model of 2:

$\mathrm{E}(\mathrm{RPBE}+\mathrm{HF}-\mathrm{PBE})=-1012.91203199$

\begin{tabular}{rrrr}
\hline 44 & -0.161744 & -0.020776 & -0.000044 \\
15 & 1.719241 & -1.111302 & -0.000067 \\
15 & 0.803375 & 2.022369 & -0.000009 \\
6 & -1.690707 & -1.577282 & 0.000361 \\
1 & -1.481562 & -2.638436 & 0.000625 \\
6 & -1.863484 & -0.748741 & 1.157676 \\
1 & -1.789954 & -1.069104 & 2.188334 \\
6 & -2.163713 & 0.574407 & 0.717031 \\
1 & -2.363702 & 1.423423 & 1.356972 \\
6 & -2.163741 & 0.574047 & -0.717403 \\
1 & -2.363782 & 1.422722 & -1.357778 \\
1 & -1.790024 & -1.070241 & -2.187848 \\
6 & -1.863527 & -0.749334 & -1.157358 \\
1 & 1.885491 & -2.520654 & 0.000018 \\
1 & -0.082342 & 3.129331 & 0.000136 \\
1 & 1.638021 & 2.491284 & -1.050534 \\
1 & 1.638208 & 2.491131 & 1.050431 \\
6 & 3.484404 & -0.578165 & 0.000086 \\
1 & 3.537094 & 0.513861 & 0.000084 \\
1 & 4.007246 & -0.947370 & 0.888424 \\
1 & 4.007417 & -0.947388 & -0.888143 \\
\hline
\end{tabular}


Model of 3:

$\mathrm{E}(\mathrm{RPBE}+\mathrm{HF}-\mathrm{PBE})=-1012.89620749$

\begin{tabular}{rrrr}
\hline 44 & 0.124987 & 0.055083 & -0.178499 \\
15 & -0.876532 & 2.034920 & 0.139618 \\
15 & -1.725519 & -1.067547 & 0.187843 \\
6 & 1.648052 & -1.584132 & -0.212254 \\
1 & 1.440419 & -2.602418 & -0.512531 \\
6 & 2.085189 & -0.533769 & -1.069765 \\
1 & 2.234170 & -0.610390 & -2.138119 \\
6 & 2.278011 & 0.635842 & -0.280698 \\
1 & 2.639701 & 1.590801 & -0.637606 \\
6 & 1.968893 & 0.298555 & 1.085275 \\
1 & 2.037869 & 0.965129 & 1.935129 \\
1 & 1.315816 & -1.623357 & 2.013409 \\
6 & 1.590731 & -1.065702 & 1.127714 \\
1 & -2.276760 & 2.098290 & 0.352257 \\
1 & -0.810369 & 3.049544 & -0.849896 \\
1 & -0.498694 & 2.861272 & 1.231902 \\
1 & -1.702653 & -2.287345 & 0.914395 \\
1 & -0.450561 & 0.266172 & -1.649048 \\
6 & -3.341487 & -0.907170 & -0.170913 \\
1 & -4.073204 & -1.631682 & 0.170178 \\
1 & -3.700726 & -0.072012 & -0.764200 \\
\hline
\end{tabular}

Transition State:

Imaginary frequency $=-2002.8072$

$\mathrm{E}(\mathrm{RPBE}+\mathrm{HF}-\mathrm{PBE})=-1012.79943012$

\begin{tabular}{rrrr}
\hline 44 & -0.093027 & 0.041794 & -0.194779 \\
15 & 0.942395 & 2.027403 & -0.050443 \\
15 & 1.666960 & -1.202744 & -0.471808 \\
6 & -1.676465 & -1.525289 & 0.013033 \\
6 & -1.443364 & -0.903720 & 1.279933 \\
6 & -1.777990 & 0.477308 & 1.138950 \\
6 & -2.304219 & 0.682591 & -0.192924 \\
6 & -2.242062 & -0.548624 & -0.872408 \\
1 & -1.529386 & -2.572945 & -0.216163 \\
1 & -1.088293 & -1.388005 & 2.179552 \\
1 & -1.745598 & 1.218480 & 1.927143 \\
1 & -2.682211 & 1.614958 & -0.590906 \\
1 & -2.526473 & -0.722659 & -1.902248 \\
1 & 1.669942 & 2.577587 & -1.139059 \\
1 & 1.932977 & 2.292110 & 0.937576 \\
1 & 0.140520 & 3.167334 & 0.209033 \\
1 & 1.527232 & -2.608436 & -0.266410 \\
6 & 2.923498 & -0.833081 & 0.712898 \\
1 & 3.512351 & -1.626961 & 1.169484 \\
1 & 3.428673 & 0.125905 & 0.611807 \\
1 & 1.436723 & -0.381316 & 1.007345 \\
\hline
\end{tabular}

\section{$\underline{\text { References }}$}

(1) Full citation for Gaussian 03 (Revision B.03): Frisch, M. J.; Trucks, G. W.; Schlegel, H. B.; Scuseria, G. E.; Robb, M. A.; Cheeseman, J. R.; Montgomery, Jr., J. A.; Vreven, T.; Kudin, K. N.; Burant, J. C. Millam, J. M.; Iyengar, S. S.; Tomasi, J.; Barone, V.; Mennucci, B.; Cossi, M.; Scalmani, G.; Rega, N.; Petersson, G. A.; Nakatsuji, H.; Hada, M.; Ehara, M.; Toyota, K.; Fukuda, R.; Hasegawa, J.; Ishida, M.; Nakajima, T.; Honda, Y.; Kitao, O.; Nakai, H.; Klene, M.; Li, X.; Knox, J. E.; Hratchian, H. P.; Cross, J. B.; Adamo, C.; Jaramillo, J.; Gomperts, R.; Stratmann, R. E.; Yazyev, O.; Austin, A. J.; Cammi, R.; Pomelli, C.; Ochterski, J. W.; Ayala, P. Y.; Morokuma, K.; Voth, G. A.; Salvador, P.; 
Dannenberg, J. J.; Zakrzewski, V. G.; Dapprich, S.; Daniels, A. D.; Strain, M. C.; Farkas, O.; Malick, D. K.; Rabuck, A. D.; Raghavachari, K.; Foresman, J. B.; Ortiz, J. V.; Cui, Q.; Baboul, A. G.; Clifford, S.; Cioslowski, J.; Stefanov, B. B.; Liu, G.; Liashenko, A.; Piskorz, P.; Komaromi, I.; Martin, R. L.; Fox, D. J.; Keith, T.; Al-Laham, M. A.; Peng, C. Y.; Nanayakkara, A.; Challacombe, M.; Gill, P. M. W.; Johnson, B.; Chen, W.; Wong, M. W.; Gonzalez, C.; Pople, J. A. Gaussian 03, Revision B.03, Gaussian, Inc., Pittsburgh PA, 2003. 